PROCEEDINGS OF THE

AMERICAN MATHEMATICAL SOCIETY

Volume 125, Number 8, August 1997, Pages 2337-2346

S 0002-9939(97)03824-0

\title{
FACTORIZATION OF HOLOMORPHIC MAPPINGS ON $C(K)$-SPACES
}

\author{
JARI TASKINEN
}

(Communicated by Theodore W. Gamelin)

\begin{abstract}
We prove a universal mapping theorem for a large class of holomorphic mappings $F$ on a $C(K)$-space, stating that $F$ can be locally written in the form $F(f)=B(1 /(1-A f))$, where $A$ and $B$ are bounded linear operators on certain Banach spaces consisting of functions on $K$, and the division is taken pointwise.
\end{abstract}

\section{INTRODUCTION}

We prove a linearization theorem for a class of holomorphic mappings $F$ on $C(K)$-spaces. We show in Theorem 3.4 that such an $F$ can be presented as a compose of bounded linear operators $A, B$ and the holomorphic mapping $H(f)(t):=$ $1 /(1-f(t))$, where $f \in U$ (the open unit ball of $C(K))$ and $t \in K$ :

$$
F(f)=B H(A f)=B\left(\frac{1}{1-A f}\right) .
$$

Here only the operator $B$ depends on $F$ so that this result can be considered as a universal mapping theorem where both the universal map $(=H \circ A)$ and also the universal space are of a very special form. The point is that the non-linearity of the universal map comes only from the simple scalar holomorphic map $z \mapsto 1 /(1-z)$.

Our result is only local: it deals only with mappings $F$ defined on open discs. Moreover, there are some unsolved problems concerning the operators $A$ and $B$. We refer to Theorem 3.4 and Remark 3.5.

Universal mapping theorems for holomorphic mappings on Banach or locally convex spaces have previously been studied for example in [Ma, Mu1, Mu2, Mu-N, $\mathrm{G}-\mathrm{G}-\mathrm{M}]$, see also $[\mathrm{R}]$.

\section{Notation. InTEGRAL HOLOMORPHiC MAPPINGS}

We denote by $\mathbf{N}$ the set $\{1,2,3, \ldots\}$ and by $\mathbf{N}_{0}$ the set $\mathbf{N} \cup\{0\}$. The closed unit interval $[0,1]$ is denoted by $I$. All Banach spaces are over the complex scalar field. The space of bounded linear operators between the Banach spaces $X$ and $Y$ is denoted by $L(X, Y)$, or by $L(X)$, if $X=Y$; the dual of $X$ is denoted by $X^{*}$. The absolutely convex hull of a subset $A$ of a Banach space is denoted by $\Gamma(A)$.

For general topology we refer to [Ku]. If $K$ is a compact metric space, we denote by $C(K)$ (resp. $\left.\ell_{\infty}(K)\right)$ the Banach space of continuous (resp. bounded), complex

Received by the editors August 16, 1995 and, in revised form, February 20, 1996.

1991 Mathematics Subject Classification. Primary 46G20; Secondary 47H99.

(C) 1997 American Mathematical Society 
valued mappings $K \rightarrow \mathbf{C}$, endowed with the sup-norm. If $K_{1}$ and $K_{2}$ are compact metric spaces and $\varphi: K_{1} \rightarrow K_{2}$ is a continuous surjection, we denote by $\varphi^{\circ}$ the linear isometry from $C\left(K_{2}\right)$ into $C\left(K_{1}\right)$ given by $\varphi^{\circ} f=f \circ \varphi$. If $\varphi^{\circ}\left(C\left(K_{2}\right)\right)$ is 1-complemented in $C\left(K_{1}\right)$, i.e., if there exists a contractive projection from $C\left(K_{1}\right)$ onto $\varphi^{\circ}\left(C\left(K_{2}\right)\right.$ ), we say that $\varphi$ admits a regular averaging operator. (Note that in this case the map $\varphi^{\circ}$ also has a contractive left inverse.) For more details we recommend the reference $[\mathrm{LT}]$, Sections II.4.h,i, and $[\mathrm{P}]$.

For complex analysis in infinite dimensional spaces we refer to [D2] and [C]. If $X$ and $Y$ are Banach spaces and $n \in \mathbf{N}$, we denote by $\mathrm{P}\left({ }^{n} X, Y\right)$ the space of continuous $n$-homogeneous polynomials $X \rightarrow Y$.

Recall that a continuous $n$-linear form $F$ on $C(K)^{n}$ is called integral, if there exists a $\mu(F) \in C\left(K^{n}\right)^{*}$ such that

$$
F\left(f_{1}, \ldots, f_{n}\right)=\left\langle\prod_{k=1}^{n} f_{k} \circ \pi_{n}^{(k)}, \mu(F)\right\rangle,
$$

where $f_{k} \in C(K), \pi_{n}^{(k)}$ is the canonical projection from $K^{n}$ onto the $k$ :th coordinate space and the product on the right-hand side is taken pointwise.

Let $U \subset C(K)$ be open and $F: U \rightarrow Y$ holomorphic. We write the Taylor series of $F$ at the point $y \in U$ as

$$
F(x)=F_{0}+\sum_{n=1}^{\infty} F_{n}^{(y)}(x-y)
$$

where $F_{0} \in Y$ and $F_{n}^{(y)} \in \mathrm{P}\left({ }^{n} C(K), Y\right)$; we denote by $\hat{F}_{n}^{(y)}$ the corresponding symmetric $n$-linear mapping.

The following definition was given in [T2].

1.1. Definition. Let $Y$ be a Banach space, let $U \subset C(K)$ be open, let $F: U \rightarrow Y$ be a holomorphic mapping, let $B \subset U$ be an open ball with center $y$ and radius $r$, and let $S \subset Y^{*}$ be a bounded subset. We say that $F$ is uniformly $(S, B)$-integral, if

$1^{\circ}$. for every $t \in S, n \in \mathbf{N}$, the $n$-linear form

$$
\left(f_{1}, \ldots, f_{n}\right) \mapsto\left\langle\hat{F}_{n}^{(y)}\left(f_{1}, \ldots, f_{n}\right), t\right\rangle
$$

is integral (write $\mu(F, n, t)$ for the corresponding element of $C\left(K^{n}\right)^{*}$ as in (1.1)), $2^{\circ}$.

$$
\|F\|_{S, B}:=\sup _{t \in S}\left\{\left|\left\langle F_{0}, t\right\rangle\right|+\sum_{n=1}^{\infty} \sup _{\substack{h \in C\left(K^{n}\right),\|h\| \leq 1}}|\langle h, \mu(F, n, t)\rangle| r^{n}\right\}<\infty
$$

and

$3^{\circ}$. the mapping

$$
t \rightarrow \sum_{n=1}^{\infty}\left\langle h_{n}, \mu(F, n, t)\right\rangle r^{n}
$$

is, for arbitrary $h_{n} \in C\left(K^{n}\right)$ with $\left\|h_{n}\right\| \leq 1$, continuous $S \rightarrow \mathbf{C}$, when $S$ is endowed with the weak* topology. 
We remark that this concept of integral holomorphic mappings does not coincide with the definition of mappings of integral holomorphy type in [D1] and [A]. Nevertheless, the definition is quite natural and gives quite a large class of holomorphic mappings.

1.2. Examples. $1^{\circ}$ The operator $f \mapsto f^{n}$ (pointwise multiplication; $n \in \mathbf{N}$ ) is uniformly integral $C(K) \rightarrow C(K)$ for every $S$ and $B$ as in Definition 1.1. We especially see that the identity operator on $C(K)$ is uniformly integral. (We refer to [T2] for the details of this and the following examples.)

$2^{\circ}$ Let $U \subset C(K)$ be the open unit ball, let $Y=C(K)$ and let $h$ be a scalar valued holomorphic mapping on the open unit disc of $\mathbf{C}$ such that its Taylor coefficients at 0 form an absolutely summable sequence. Then the map $(H f)(t):=h(f(t))$, $f \in U, t \in K$, is uniformly $(K, U)$-integral on $U$; here the set $K$ is identified in the canonical way with a subset of $C(K)^{*}$.

$3^{\circ}$ Denote by $U \subset C(I)$ the open unit ball. If $F_{n}: I \times I^{n} \rightarrow \mathbf{C}$ is for all $n \in \mathbf{N}_{0}$ a continuous function satisfying $\sum_{n=0}^{\infty}\left\|F_{n}\right\|_{C\left(I^{n+1}\right)}<\infty$, then the holomorphic integral operator

$$
f \mapsto \sum_{n=0}^{\infty} \int_{I^{n}} F_{n}\left(\cdot, s_{1}, \ldots, s_{n}\right) f\left(s_{1}\right) \ldots f\left(s_{n}\right) d s
$$

where $s=\left(s_{1}, \ldots, s_{n}\right)$, is uniformly $(I, U)$-integral $U \rightarrow C(I)$.

\section{Preliminary Results}

In this section we present some results necessary for the proof of the main result.

In the following universal mapping theorem we denote by $K$ a compact metric uncountable space and by $U$ the open unit ball of $C(K)$. The set $K$ is also considered as a subset of $C(K)^{*}$ : for every $t \in K$ there corresponds the point evaluation $\delta_{t}: f \mapsto f(t), f \in C(K)$. This identification is a homeomorphism, when $C(K)^{*}$ is endowed with the weak ${ }^{*}$-topology.

2.1. Theorem. There exists a universal holomorphic mapping $\psi: U \rightarrow C(K)$ such that for every uniformly $(K, U)$-integral holomorphic $F: U \rightarrow C(K)$ there exists $B_{F} \in L(C(K))$ such that the following diagram commutes:

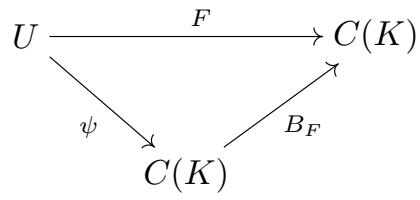

This result was proved in [T2], Theorem 2.1.

For the proof of the main theorem of this paper we shall need the following

Remark. Having a look at the proof of Theorem 2.1 of [T2] (especially (2.2) there) one easily verifies that

$$
\psi(f)=f_{0}+\sum_{n=1}^{\infty} \prod_{k=1}^{n} \psi_{n}^{(k)} f
$$

where $f_{0}$ is an element of $C(K), \psi_{n}^{(k)} \in L(C(K))$ and $\left\|\psi_{n}^{(k)}\right\| \leq 1$ for all $n$ and $k$. 
2.2. Lemma. Let $n \in \mathbf{N}$, let $J$ be a finite set and let $\left(x_{j}\right)_{j \in J}$ be a sequence of complex numbers satisfying $\left|x_{j}\right|<1$. For all sequences of complex numbers $\left(\lambda_{j}\right)_{j \in J}$

$$
\sup _{t \in[0,1]}\left|\sum_{j \in J} \lambda_{j} \frac{1}{1-\mathrm{e}^{i 2 t \pi} x_{j}}\right| \geq\left|\sum_{j \in J} \lambda_{j} x_{j}^{n}\right| .
$$

Proof. Using the Taylor series of the analytic function $z \mapsto 1 /(1-z),|z|<1$, we easily get

$$
\int_{0}^{1} \mathrm{e}^{-i 2 n t \pi} \sum_{j \in J} \lambda_{j} \frac{1}{1-\mathrm{e}^{i 2 t \pi} x_{j}} d t=\sum_{j \in J} \lambda_{j} x_{j}^{n}
$$

This implies the inequality (2.2).

2.3. Proposition. There exist a strictly increasing sequence $(\tau(t))_{t=0}^{\infty}, \tau(t) \in \mathbf{N}_{0}$, $\tau(0)=1$, and, for every $m \in \mathbf{N}, n \in \mathbf{N}_{0}$, complex numbers $a_{m, n}$ and $b_{m, n}$ such that the following holds (convention: $0^{0}=1$ ).

For every $m \in \mathbf{N}, n \in \mathbf{N}_{0}$, we have $\left|a_{m, n}\right|<1,\left|b_{m, n}\right| \leq e^{7}$.

For all $z \in \mathbf{C},|z|<1$, for all $n \in \mathbf{N}_{0}$,

$$
\sum_{t \in \mathbf{N}_{0}} \sum_{m=\tau(t)}^{\tau(t+1)-1} \sum_{k \in \mathbf{N}_{0}} b_{m, n} a_{m, n}^{k} z^{k}=z^{n},
$$

and

$$
\sum_{t \in \mathbf{N}_{0}} \sum_{m=\tau(t)}^{\tau(t+1)-1} b_{m, 0}=1 .
$$

This result is contained in Theorem 9 of [T4].

\section{Holomorphic mapping as a COMPOSE of Linear operators AND A SCALAR HOLOMORPHIC FUNCTION}

Let $U \subset C(K)$ be the open unit ball and let $r>\mathrm{e}$. In this section we show that a uniformly $(K, r U)$-integral holomorphic $F: r U \rightarrow C(K)$ can be presented as a product of linear operators $A, B$ and the mapping $H: f(t) \mapsto 1 /(1-f(t)), \quad f \in U$, $t \in K$. More precisely, we show that the equality

$$
F(f)=B H(A f)
$$

holds for $f \in U$.

There are two major difficulties. First, one needs to solve at least approximately an infinite system of polynomially nonlinear equations. The solution is presented in detail in the paper [T4] and only the result is mentioned here; see Proposition 2.3. The second difficulty is to make the operators $A$ and $B$ well defined and continuous. We are not able to solve this problem in the optimal way. Accordingly, $A$ becomes a bounded operator in the sup-norm, but the functions $A f$, where $f \in C(K)$, need not be continuous everywhere in $K$. (However, the discontinuity is in some sense only "mild".) We are in general able to define the operator $B$ only in the closed linear span of $H \circ A$, not in the whole space $C(K)$ (or $\left.\ell_{\infty}(K)\right)$. Finally, we need to assume that the given map $F$ is holomorphic in $r U$, not only in $U$ (see above). We refer to Remark 3.5 for some explanations. 
3.1. Definitions. We choose for every $n, j \in \mathbf{N}_{0}, 1 \leq j \leq 2^{n}$, a closed subinterval $I_{n, j} \subset I=[0,1]$ such that $I_{n, j} \cap I_{n^{\prime}, j^{\prime}}=\emptyset$, if $n \neq n^{\prime}$ or $j \neq j^{\prime}$, and such that for every $\varepsilon>0$ the interval $[0,1-\varepsilon]$ contains only finitely many intervals $I_{n, j}$.

We choose for every $n, j$ a continuous surjection $\varphi_{n, j}: I_{n, j} \rightarrow I^{3}$ with a regular averaging operator. (See [H], Theorem 2.2., also [T3], Theorem 2.) We denote by $\hat{\varphi}_{n, j}$ a contractive left inverse of $\varphi_{n, j}^{\circ}$.

We fix for every $n, j$ a Borsuk-Kakutani extension operator $E_{n, j}: C\left(I_{n, j}\right) \rightarrow$ $C(I)$ such that $\operatorname{supp} E_{n, j} f \cap \operatorname{supp} E_{n^{\prime}, j^{\prime}} g=\emptyset$ for all $f, g$, if $n \neq n^{\prime}$ or $j \neq j^{\prime}$. (See [LT], Theorem II.4.14.; since the sets $I_{n, j}$ have mutually disjoint open neighbourhoods for different indices $n, j$, a simple trick shows that our requirement for the disjointness of the sets $\operatorname{supp} E_{n, j} f$ can be satisfied.) We fix some disjoint closed intervals $J_{n, j} \supset I_{n, j}, J_{n, j} \subset I$, such that $\operatorname{supp} E_{n, j} f \subset J_{n, j}$ for all $f \in C\left(I_{n, j}\right)$.

For every $m \in \mathbf{N}$ and $n, j$ we denote by $K_{m, n, j}$ a subspace of $I^{3}$ of the form $I_{n, j} \times\left\{s_{2}\right\} \times\left\{s_{3}\right\}$, where the numbers $s_{2}, s_{3} \in I$ are chosen such that

$$
s_{2} e^{i 2 \pi s_{3}}=a_{m, n} .
$$

Here $a_{m, n}$ is as in Proposition 2.3. We denote by $\eta_{m, n, j}: I_{n, j} \rightarrow K_{m, n, j}$ the homeomorphism $t \mapsto t \times\left\{s_{2}\right\} \times\left\{s_{3}\right\}$.

3.2. Lemma. Use the notation of Proposition 2.3 and Definition 3.1, and fix some indices $n \in \mathbf{N}_{0}, j \in \mathbf{N}, 1 \leq j \leq 2^{n}$. Let $\alpha \in L\left(C\left(I^{3}\right)\right)$ be a contraction. Define $\alpha_{n}^{(j)} \in L\left(C\left(I^{3}\right)\right)$ by

$$
\left(\alpha_{n}^{(j)} f\right)\left(t_{1}, t_{2}, t_{3}\right)=t_{2} e^{i 2 \pi t_{3}}\left(E_{n, j} \varphi_{n, j}^{\circ} \alpha f\right)\left(t_{1}\right)
$$

where $\left(t_{1}, t_{2}, t_{3}\right) \in I^{3}$.

$1^{\circ}$. We have $\left\|\alpha_{n}^{(j)} f\right\| \leq\|\alpha f\|$ for all $f$.

$2^{\circ}$. For every $f \in H\left(\alpha_{n}^{(j)}(U)\right)$, where $U \subset C\left(I^{3}\right)$ is the open unit ball, the sum

$$
\beta_{n}^{(j)} f:=\sum_{t=0}^{\infty} \sum_{m=\tau(t)}^{\tau(t+1)-1} b_{m, n} \hat{\varphi}_{n, j} \eta_{m, n, j}^{\circ} f
$$

converges pointwise in $I^{3}$ and defines a continuous mapping from $H\left(\alpha_{n}^{(j)}(U)\right)$ into $C\left(I^{3}\right)$ which can be extended as a bounded linear operator to the subspace $\overline{\operatorname{sp} H\left(\alpha_{n}^{(j)}(U)\right)}$. Denoting the extension again by $\beta_{n}^{(j)}$ we have $\left\|\beta_{n}^{(j)}\right\| \leq 1$.

$3^{\circ}$. We can define, without increasing the norm of $\beta_{n}^{(j)}$,

$$
\beta_{n}^{(j)} \sum_{k} \lambda_{k} H\left(f_{k}+g_{k}\right)=\beta_{n}^{(j)} \sum_{k} \lambda_{k} H\left(f_{k}\right)
$$

for all finite sequences $\left(\lambda_{k}\right) \subset \mathbf{C},\left(f_{k}\right) \subset \alpha_{n}^{(j)}(U)$ and $\left(g_{k}\right) \subset \ell_{\infty}\left(I^{3}\right)$ such that $\left\|g_{k}\right\|<1, \operatorname{supp}\left(g_{k}\right) \cap J_{n, j} \times I^{2}=\emptyset$.

$4^{\circ}$. We have for $n \geq 1$

$$
\beta_{n}^{(j)} H\left(\alpha_{n}^{(j)} f\right)=(\alpha f)^{n}
$$

for all $f \in U$, and $\beta_{0}^{(1)} H(0)=1$.

Proof. $1^{\circ}$. This is clear.

$2^{\circ}$. Assume that $f \in H\left(\alpha_{n}^{(j)}(U)\right), f=H\left(\alpha_{n}^{(j)} g\right)$ for some $g \in U$. Because of the definition of $K_{m, n, j}$ we have for every $m \in \mathbf{N}_{0}$

$$
f \circ \eta_{m, n, j}=H\left(a_{m, n} \varphi_{n, j}^{\circ} \alpha g\right)=\varphi_{n, j}^{\circ} H\left(a_{m, n} \alpha g\right) .
$$


Hence,

$$
\beta_{n}^{(j)} f=\sum_{t=0}^{\infty} \sum_{m=\tau(t)}^{\tau(t+1)-1} b_{m, n} H\left(a_{m, n} \alpha g\right)=\sum_{t=0}^{\infty} \sum_{m=\tau(t)}^{\tau(t+1)-1} b_{m, n} \sum_{k=0}^{\infty}\left(a_{m, n} \alpha g\right)^{k},
$$

and Proposition 2.3 implies the desired pointwise convergence of (3.4). Moreover, by Proposition 2.3 and (3.6),

$$
\beta_{n}^{(j)} f=(\alpha g)^{n}
$$

so that $\beta_{n}^{(j)} f \in C\left(I^{3}\right)$.

We extend $\beta_{n}^{(j)}$ linearly to $\operatorname{sp} H\left(\alpha_{n}^{(j)}(U)\right)$ and prove that the extension is a bounded operator. To this end let $J \subset \mathbf{N}$ be a finite sequence, let for every $k \in J$ the functions $f_{k} \in U$ and the complex numbers $\lambda_{k}$ be arbitrary. We apply Lemma 2.2 to get the estimate

$$
\begin{aligned}
& \quad\left\|\beta_{n}^{(j)} \sum_{k \in J} \lambda_{k} H\left(\alpha_{n}^{(j)} f_{k}\right)\right\|=\left\|\sum_{k \in J} \lambda_{k}\left(\alpha f_{k}\right)^{n}\right\| \\
& \leq \sup _{t_{2} \in I, t_{3} \in I} \sup _{t \in I^{3}}\left|\sum_{k \in J} \lambda_{k} \frac{1}{1-t_{2} e^{i 2 \pi t_{3}}\left(\alpha f_{k}\right)(t)}\right| .
\end{aligned}
$$

Recall that each $\varphi_{n, j}$ is a surjection and each $E_{n, j}$ is an extension operator. Hence, (3.8) is not greater than

$$
\begin{aligned}
\sup _{t_{2} \in I, t_{3} \in I} \sup _{t \in I} \mid & \sum_{k \in J} \lambda_{k} \frac{1}{1-t_{2} e^{i 2 \pi t_{3}}\left(E_{n, j} \varphi_{n, j}^{\circ} \alpha f_{k}\right)(t)} \mid \\
& =\left\|\sum_{k \in J} \lambda_{k} H\left(\alpha_{n}^{(j)} f_{k}\right)\right\| .
\end{aligned}
$$

This proves the boundedness of $\beta_{n}^{(j)}$ in $E:=\overline{\operatorname{sp} H\left(\alpha_{n}^{(j)}(U)\right)}$ and the desired norm estimate.

$3^{\circ}$. The operator $\beta_{n}^{(j)}$ is extended above to $E$. We have $\operatorname{supp} f \subset J_{n, j} \times I^{2}$ for all $f \in \alpha_{n}^{(j)}\left(C\left(I^{3}\right)\right)$. Hence, for all $\lambda_{k} \in \mathbf{C}, f_{k} \in \alpha_{n}^{(j)}(U)$ and $g_{k} \in \ell_{\infty}\left(I^{3}\right)$ such that $\left\|g_{k}\right\|<1$ and $\operatorname{supp} g_{k} \cap J_{n, j} \times I^{2}=\emptyset$,

$$
\begin{gathered}
\left\|\beta_{n}^{(j)} \sum_{k} \lambda_{k} H\left(f_{k}+g_{k}\right)\right\|=\left\|\beta_{n}^{(j)} \sum_{k} \lambda_{k} H\left(f_{k}\right)\right\| \\
\leq\left\|\sum_{k} \lambda_{k} H\left(f_{k}\right)\right\| \leq\left\|\sum_{k} \lambda_{k} H\left(f_{k}+g_{k}\right)\right\| .
\end{gathered}
$$

(The assumption on the supports is used to get the last inequality.)

$4^{\circ}$. Follows from (3.7) and (2.4).

3.3. Lemma. Let $\left(\left(A_{n}^{(j)}\right)_{j=1}^{2^{n}}\right)_{n=1}^{\infty}$ be a sequence of linear contractions $C\left(I^{3}\right) \rightarrow$ $C\left(I^{3}\right)$ and let $\varepsilon>0$. Let $\psi: U \rightarrow C\left(I^{3}\right)$ be the holomorphic mapping

$$
\psi(f)(t):=f_{0}(t)+\sum_{n=1}^{\infty} \sum_{j=1}^{2^{n}} \varepsilon_{n}^{(j)}\left(A_{n}^{(j)} f\right)(t)^{n}
$$

where $\varepsilon_{n}^{(j)} \in \mathbf{C},\left|\varepsilon_{n}^{(j)}\right| \leq(2+\varepsilon)^{-n}$ and $f_{0} \in C\left(I^{3}\right)$ is fixed. 
There exist linear operators $A \in L\left(C\left(I^{3}\right), \ell_{\infty}\left(I^{3}\right)\right)$ and $B_{1} \in L\left(E, C\left(I^{3}\right)\right)$, where $E \subset \ell_{\infty}\left(I^{3}\right)$ is the closed linear span of $H(A(U))$, such that

$$
\psi(f)=B_{1} H(A f)
$$

for all $f \in U$.

Proof. We use the notations of Proposition 2.3, Definition 3.1 and Lemma 3.2. For every $n \in \mathbf{N}_{0}$ and $j=1, \ldots, 2^{n}$ we choose the operators $\alpha_{n}^{(j)}$ and $\beta_{n}^{(j)}$ as in Lemma 3.2 , taking $\alpha=A_{n}^{(j)}$ and $\alpha=0$ in the case $n=0, j=1$. We define

$$
\begin{aligned}
& A=\sum_{n, j} \alpha_{n}^{(j)}, \\
& B_{1}=\beta_{0}^{(1)}+\sum_{n \in \mathbf{N}} \sum_{j=1}^{2^{n}} \varepsilon_{n}^{(j)} \beta_{n}^{(j)} .
\end{aligned}
$$

That $A \in L\left(C\left(I^{3}\right), \ell_{\infty}\left(I^{3}\right)\right)$ follows from $1^{\circ}$ of Lemma 3.2 and from the assumption on the supports of the functions $E_{n, j} f$ (Definition 3.1). The boundedness of $B_{1}$ follows from the facts that ||$\beta_{n}^{(j)} \| \leq 1$ and $\left|\varepsilon_{n}^{(j)}\right| \leq(2+\varepsilon)^{-n}$ for every $n, j$, and from $3^{\circ}$ of Lemma 3.2. The statements $3^{\circ}$ and $4^{\circ}$ of Lemma 3.2 yield, for $f \in U$,

$$
\begin{aligned}
B_{1} H(A f) & =f_{0} \beta_{0}^{(1)} H(0)+\sum_{n \in \mathbf{N}} \sum_{j=1}^{2^{n}} \varepsilon_{n}^{(j)} \beta_{n}^{(j)} H\left(\alpha_{n}^{(j)} f\right) \\
& =f_{0}+\sum_{n \in \mathbf{N}} \sum_{j=1}^{2^{n}} \varepsilon_{n}^{(j)}\left(A_{n}^{(j)} f\right)^{n} .
\end{aligned}
$$

In the following theorem we denote by $K$ a compact metrizable uncountable space which has a closed subspace homeomorphic to $I$ and which is a Peano space (i.e. a continuous image of $I$ ), and by $U$ the open unit ball of $C(K)$. Recall that for example every connected compact manifold is this kind of space $K$.

3.4. Theorem. Let $r>e$ and let $F: r U \rightarrow C(K)$ be a uniformly $(K, r U)$-integral holomorphic mapping. There exist linear operators $A \in L\left(C(K), \ell_{\infty}(K)\right)$ and $B \in$ $L(E, C(K))$, where $E \subset \ell_{\infty}(K)$ is the closed linear span of $H(A(U))$, such that

$$
F(f)=B H(A f)=B\left(\frac{1}{1-A f}\right)
$$

for all $f \in U$.

Proof. $1^{\circ}$. We first consider the case $K=I^{3}$. One easily verifies that the mapping $G: U \rightarrow C\left(I^{3}\right), G(x):=F(r x)$, is uniformly $(K, U)$-integral. We apply Theorem 2.1 to write $G=B_{G} \circ \psi_{G}$, where $B_{G} \in L\left(C\left(I^{3}\right)\right)$,

$$
\psi_{G}(f)=f_{0}+\sum_{n=1}^{\infty} \prod_{k=1}^{n} \psi_{n}^{(k)} f
$$

and $\left\|\psi_{n}^{(k)}\right\| \leq 1$ for all $n$ and $k$ (see (2.1)). We get the representation $F=B_{G} \circ \psi$, where

$$
\psi(f)=f_{0}+\sum_{n=1}^{\infty} r^{-n} \prod_{k=1}^{n} \psi_{n}^{(k)} f .
$$


For all $n \in \mathbf{N}$, the polarization formula ([D2], Theorem 1.5) implies the following equality for all complex numbers $\lambda_{k}, k=1, \ldots, n$ :

$$
\prod_{k=1}^{n} \lambda_{k}=\frac{1}{n ! 2^{n}} \sum_{\varepsilon_{j}= \pm 1} \varepsilon_{1} \ldots \varepsilon_{n}\left(\sum_{k=1}^{n} \varepsilon_{k} \lambda_{k}\right)^{n} .
$$

Applying this we get

$$
\begin{aligned}
& \psi(f)=f_{0}+\sum_{n \in \mathbf{N}} r^{-n} 2^{-n}(n !)^{-1} n^{n} \sum_{\varepsilon_{j}= \pm 1} \varepsilon_{1} \ldots \varepsilon_{n}\left(n^{-1} \sum_{k=1}^{n} \varepsilon_{k} \psi_{n}^{(k)} f\right)^{n} \\
& =f_{0}+\sum_{n \in \mathbf{N}} e^{-n}(n !)^{-1} n^{n} \sum_{\varepsilon_{j}= \pm 1}(2 r / e)^{-n} \varepsilon_{1} \ldots \varepsilon_{n}\left(n^{-1} \sum_{k=1}^{n} \varepsilon_{k} \psi_{n}^{(k)} f\right)^{n} .
\end{aligned}
$$

We have

$$
e^{-n}(n !)^{-1} n^{n} \leq 1,
$$

since $(n !)^{-1} n^{n}$ is the $n$th term in the Taylor series of $e^{n}$. Hence (3.16) yields a representation for $\psi$ which satisfies the assumptions of Lemma 3.3; in particular, $A_{n}^{(j)}:=n^{-1} \sum_{k=1}^{n} \varepsilon_{k} \psi_{n}^{(k)}$. Let $A$ and $B_{1}$ be as in Lemma 3.3. Setting

$$
B=B_{G} B_{1}
$$

gives the the desired factorization of $F$ in the case $K=I^{3}$.

$2^{\circ}$. Let $K$ be arbitrary. We first choose continuous surjections $\varphi: K \rightarrow I^{3}$ and $\varrho: I^{3} \rightarrow K$ with regular averaging operators. Concerning $\varrho$, it is enough to take a retraction $I^{3} \rightarrow I$ and compose it with a continuous surjection $I \rightarrow K$ having a regular averaging operator (see [H], Theorem 2.2., or [T3], Theorem 2.). The map $\varphi$ can be found by composing a retraction from $K$ onto a subspace homeomorphic to $I$ (recall that $I$ is an absolute retract space), with a continuous surjection $I \rightarrow I^{3}$ having a regular averaging operator. We denote a contractive left inverse of $\varrho^{\circ}$ by $\varrho$. Taking a (usually discontinuous) right inverse $\varphi^{-1}$ of $\varphi$ one can define a contractive left inverse $\hat{\varphi}$ for $\varphi^{\circ}$ by $\varphi^{-10} \in L\left(\ell_{\infty}(K), \ell_{\infty}\left(I^{3}\right)\right)$.

If $F$ is as in the assumption, it follows in a straightforward way from Definition 1.1 that $G:=\varrho^{\circ} \circ F \circ \varrho$ is a uniformly $\left(I^{3}, V\right)$-integral holomorphic mapping $V \rightarrow C\left(I^{3}\right)$, where $V \subset C\left(I^{3}\right)$ is the open unit ball. By part $1^{\circ}$ of the proof we find bounded linear operators $A_{G}$ and $B_{G}$ as in (3.13) such that

$$
G(f)=B_{G} H\left(A_{G} f\right)
$$

for $f \in V$. We get for $f \in U$

$$
\begin{gathered}
F(f)=\hat{\varrho} \varrho^{\circ} F\left(\varrho \varrho^{\circ} f\right)=\hat{\varrho} B_{G} H\left(A_{G} \varrho^{\circ} f\right) \\
=\varrho B_{G} \hat{\varphi} \varphi^{\circ} H\left(A_{G} \varrho^{\circ} f\right)=\hat{\varrho} B_{G} \hat{\varphi} H\left(\varphi^{\circ} A_{G} \varrho^{\circ} f\right),
\end{gathered}
$$

so that setting $B=\hat{\varrho} B_{G} \hat{\varphi}$ and $A:=\varphi^{\circ} A_{G} \varrho^{\circ}$ yields the result. We leave the details to the reader.

3.5. Remarks. $1^{\circ}$. In the case $K=I^{3}$ the elements of $A(C(K))$ can be discontinuous only in the subset $\{1\} \times I^{2}$ of the boundary of $I^{3}$. In the case of general $K$ the discontinuity may be more serious; it depends on the choice of the mapping $\varphi$ above. 
The explanation for the discontinuity lies in the coefficients $\varepsilon_{k}$ in the polarization formula (see (3.16)): they cause the space $A(C(K))$ to necessarily contain functions which oscillate infinitely often in $K$ with a constant amplitude. The space $C(K)$ does not contain such elements.

$2^{\circ}$. By the extension property of the space $\ell_{\infty}(K)$ it is always possible to extend $B$ as a bounded operator $\ell_{\infty}(K) \rightarrow \ell_{\infty}(K)$. (See [LT1], Proposition 2.f.2.(iii).) In some cases it is possible to extend $B$ even as a bounded operator $\overline{E+C(K)} \rightarrow C(K)$ where $\overline{E+C(K)}$ is considered as a closed subspace of $\ell_{\infty}(K)$; see Theorem 3.6 below.

$3^{\circ}$. There is a natural explanation for the constant $r>e>1$. It comes (modulo an $\varepsilon>0$ ) basically from the fact that we cannot avoid the use of the polarization formula in (3.16). A related fact is that the "uniformly integral norm" (1.3) somehow measures the symmetric multilinear mappings in the Taylor series of the given $F$, whereas the representation (3.13) is more like a "polynomial of an infinite degree"; compare to [D2], Theorem 1.7.

3.6. Theorem. Let $r, K, U$ and $F$ be as in Theorem 3.4. Assume that for every $n \in \mathbf{N}$ the linear operator

$$
T(F, n) \in L\left(C\left(K^{n}\right), C(K)\right), \quad(T(F, n) f)(t)=\langle f, \mu(F, n, t)\rangle \quad \text { for } t \in K
$$

where $\mu(F, n, t)$ is as in Definition $1.1,1^{\circ}$, is compact. Then the operator $B$ of Theorem 3.4 can be extended to an element of $L(\overline{E+C(K)}, C(K))$ or $L\left(\ell_{\infty}(K), C(K)\right)$, where $\overline{E+C(K)}$ is considered as a closed subspace of $\ell_{\infty}(K)$.

Proof. The assumption (3.19) implies that $B_{F}$ in the universal mapping Theorem 2.1 is compact. (The reader has to verify this from the proof of Theorem 2.1. of [T2], especially (2.4) and (2.5) there.) Hence, also the operator $B$ is compact, see (3.12) and (3.17) etc. The result follows now from the extension properties of $\mathrm{L}_{\infty}$-spaces for compact operators, see [LT], Theorem II.5.25.2.

\section{REFERENCES}

[A] R. Aron, Holomorphy types for open subsets of a Banach space, Studia Math. 45 (1973), 273-289. MR 49:5838

[C] S. B. Chae, Calculus and holomorphy in normed spaces, Marcel Dekker, New York, 1985.

[D1] S. Dineen, Holomorphy types on Banach spaces, Studia Math. 39 (1971), 241-288. MR 46:3837

[D2] S. Dineen, Complex analysis in locally convex spaces, North Holland Mathematics Studies, vol. 57, 1981. MR 84b:46050

[G-G-M] P. Galindo, D. Garcia, M. Maestre, Holomorphic mappings of bounded type, J. Math. Anal. Appl. 166.1. (1992), 236-246. MR 94b:46069

$[\mathrm{H}] \quad$ B. Hoffmann, An injective characterization of Peano spaces, Topol. and Appl. 11 (1980), 37-46. MR 80m:54049

$[\mathrm{Ku}] \quad$ K. Kuratowski, Topology, vol. I, Academic Press, New York and London, 1966. MR 36: 840

[LT] J. Lindenstrauss, L. Tzafriri, Classical Banach Spaces, Springer Lecture Notes in Mathematics 338 (1973). MR 54:3344

[LT1] J. Lindenstrauss, L. Tzafriri, Classical Banach Spaces I., Springer, Berlin-HeidelbergNew York, 1977.

[Ma] P. Mazet, Analytic sets in locally convex spaces, North-Holland Mathematics Studies, vol. 120, North-Holland, Amsterdam, 1986.

[Mu1] J. Mujica, Linearization of bounded holomorphic mappings on Banach spaces, Trans. Amer. Math. Soc. 324 (1991), 867-887. MR 91h:46088 
[Mu2] J. Mujica, Linearization of holomorphic mappings of bounded type, Progress in Functional Analysis (K. D. Bierstedt, J. Bonet, J. Horvath, M. Maestre, eds.), vol. 170, North-Holland Mathematics Studies, 1992, pp. 149-162. MR 93k:46037

$[\mathrm{Mu}-\mathrm{N}]$ J. Mujica, L. Nachbin, Linearization of holomorphic mappings on locally convex spaces, J. Math. Pures Appl. 71 (1992), 543-560. MR 93k:46038

[P] A. Pełczyński, Linear extensions, linear averaging and application to linear topological classification of spaces of continuous functions, Rozprawy Matematyczne 58 (1968). MR 37:3335

[R] R. A. Ryan, Applications of topological tensor products to infinite dimensional holomorphy, Ph.D thesis, Trinity College, Dublin, 1980.

[T1] J. Taskinen, An application of averaging operators to multilinearity, Math. Ann. 297 (1993), 567-572. MR 94j:46031

[T2] J. Taskinen, Linearization of holomorphic mappings on $C(K)$-spaces, Isr. J. Math. 92 (1995), 1-3, 207-219. MR 96h:46065

[T3] J. Taskinen, A continuous surjection from the unit interval onto the unit square, Rev.Mat.Univ. Complutense Madrid 6.1 (1993), 101-120. MR 94i:46023

[T4] An infinite polynomially non-linear system of equations, J. Math. Anal. Appl. 200 (1996), 591-613. CMP 96:13

Department of Mathematics, P.O. Box 4 (Hallituskatu 15), Fin-00014 University of Helsinki, Finland

E-mail address: Jari.Taskinen@Helsinki.Fi 